\title{
Preservation of facial nerve functions during vestibular schwannoma surgery: outcome analysis
}

\author{
Ashraf Mohamed Farid ${ }^{*}$ (D) and Ahmed Rizk Elkholy
}

\begin{abstract}
Background: The goal of modern vestibular schwannoma surgery is not only to complete tumor removal but also to preserve functions. Cerebellopontine angle surgery carries a significant risk of impairing facial nerve function especially with large masses. Avoidance of facial nerve injury postoperatively requires preoperative and intraoperative identification and hence the role of preoperative imaging and intraoperative monitoring.

Objectives: To evaluate the impact of identification of facial nerve preoperatively and intraoperative neurophysiological monitoring on the preservation of facial nerve function.

Study design: This is a retrospective clinical case series.

Patients and methods: This study was conducted on 32 patients operated upon for excision of vestibular schwannoma. All had hearing loss but intact facial nerve (House-Brackmann (HB) grade I). Fast imaging employing steady-state acquisition was used preoperatively to predict the location of the facial nerve. Intraoperative monitoring was used for all patients. Patients were followed up for 24 months.

Results: House-Brackmann grade I was achieved in $68.75 \%$ of patients. Grade VI was achieved in $12.5 \%$ of patients. Near-total removal was achieved in $62.5 \%$ of patients, and subtotal removal was achieved in $37.5 \%$. The higher percentage of normal facial function postoperatively (HB grade I) was associated with subtotal resection and soft consistency of the neoplasm.

Conclusion: Facial preservation requires preoperative prediction of the location of the nerve in relation to the capsule, extra-arachnoidal dissection during surgery, and subtotal resection if intraoperative monitoring predicts a high risk.
\end{abstract}

Keywords: Vestibular schwannoma surgery, Facial nerve preservation

\section{Introduction}

Preservation of facial nerve injury during resection of a VS is a primary aim of the skull base surgeon. Facial paralysis has potentially devastating functional and psychological consequences for patients. The importance of intraoperative monitoring especially during resection of vestibular schwannomas comes from the anatomical fact that the facial nerve, from the brainstem to the internal auditory canal, has no epineurium [1-3].

\footnotetext{
* Correspondence: Aschraffarid@gmail.com

Neurosurgery Department, Tanta University, Tanta, Egypt
}

\section{Patients and methods \\ Patient population}

This is a retrospective clinical case series conducted on 22 females and 10 males (total 32 patients) in the Department of Neurosurgery, Tanta University Hospitals. Those patients were operated upon for excision of vestibular schwannoma in the period between February 2016 and November 2018. The main symptoms were hearing loss, tinnitus, and vestibular dysfunctions. All patients had total hearing loss, proved by both clinical examination and audio-vestibular studies. All patients had intact facial nerve preoperatively (House-Brackmann grade I) [4]. All patients' data, diagnosis, and treatment outcomes are confidentially kept private, and patients 
are presented by specific codes. All patients were consented based upon the conclusion of the study of Kondziolka et al. [5].

An approval from the research ethics committee of the Faculty of Medicine, Tanta University (reference number: 32806), was obtained on 26 December 2018. Furthermore, being a retrospective study, patients' consents for participation and for publication were not applicable. Additionally, the co-authors had neither financial nor non-financial competing interest to report.

\section{Imaging}

MRI with IV gadolinium was done preoperatively for all patients. All had radiological signs that are highly suggestive of acoustic neoplasms. The size of the neoplasms in all patients was of grade IV, i.e., more than $3 \mathrm{~cm}$, displaced both the brainstem and fourth ventricle [6]. Depending upon the presence of a CSF signal between the nerve and the neoplasm [7], fast imaging employing steady-state acquisition (FIESTA) sequence MRI was carried out for all patients to predict the possible location of the facial nerve in relation to the capsule of neoplasm where in two of our patients, the FIESTA was predictive of posteroinferior location (Fig. 1).

\section{Surgical procedures}

Prior to the definitive surgery, anesthetic considerations were taken, aiming at optimum cerebellar relaxation. Propofol was the preferred anesthetic agent; it does not interfere with monitoring techniques. A short-acting muscle relaxant sufficient for intubation was given $[8,9]$. Park-bench position was the preferred position for all of the patients

\section{Electrode positioning}

Two electrodes were used subdermally for the orbicularis oculi muscle (at the lateral aspect of the orbit), another two electrodes for the orbicularis oris muscle at the corner of the mouth, and one electrode at the forehead. These steps, together with the setup of the device settings, were carried out by the electrophysiology team (Inomed ${ }^{\bullet}$ Medizintechnik GmbH, München, Germany).

Surgical procedure was carried out as classic suboccipital craniectomy. After durotomy and tumor identification, the stimulating electrode was applied to the posterior capsule [10]. We started by pulsed stimulation using monopolar electrode, and at the same time, the electrophysiology team enabled an intensity of $0.05 \mathrm{~mA}$ as a baseline threshold. Mapping of the facial nerve was thus carried out.

\section{Interpretation of the neurophysiological monitoring responses}

At the first stage, the action potential response of the stimulated muscle(s) was recorded (Fig. 2). The second step was that we started by central debulking. During the procedure of central debulking then separation of the capsule from the nerve or from the brainstem, we were notified by the electrophysiology team if there are recorded responses (train or burst types) (Fig. 3), either

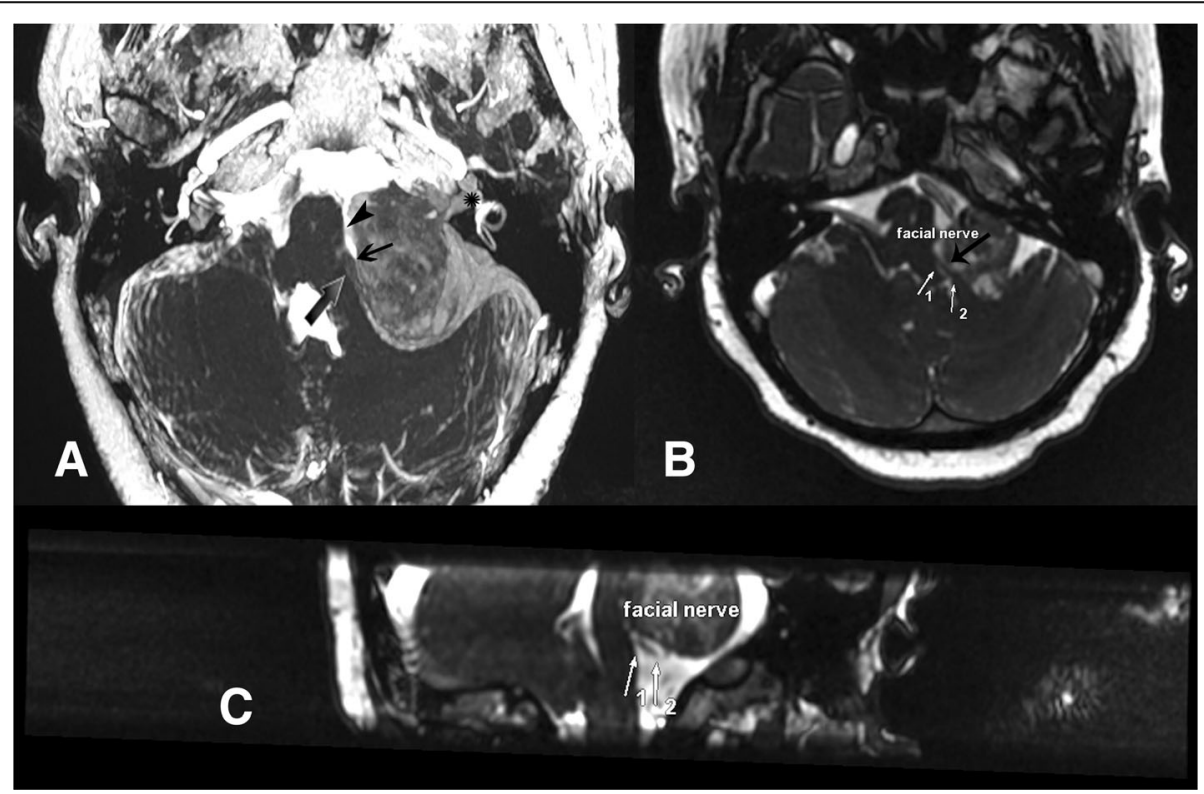

Fig. 1 FIESTA study showing the posterior location of the facial nerve. a Axial image where the arrowhead points to the CSF signal between the medial surface of the tumor and the brainstem. Arrows point to the nerve and its posterior direction. $\mathbf{b}$ Axial image for another patient where white and black arrows point to the nerve and its direction. c Coronal reconstruction showing the posteroinferior location. The other side showing the cerebellar tonsils 


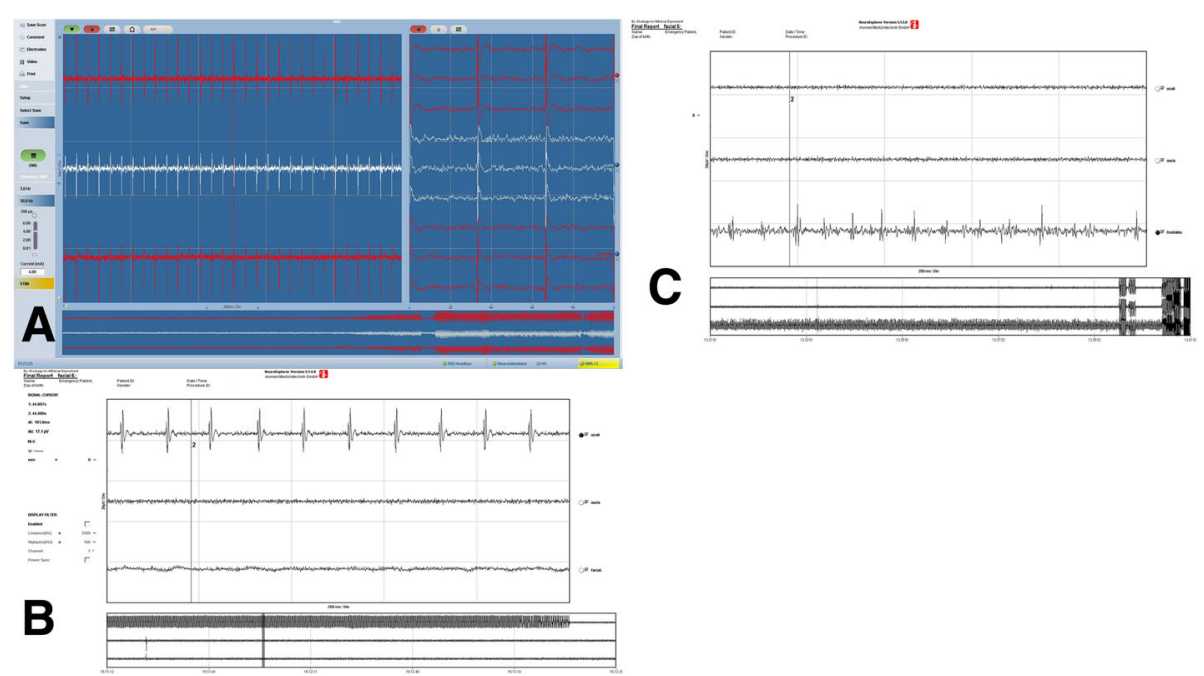

Fig. 2 Action potential for facial muscles: single or multiple. a Action Potential of the three muscles [occuli, oris and frontalis]. b Action Potential of single facial muscle [occuli]. c Action Potential of single facial muscle [frontalis]

from individual muscle or all muscles. Immediately, we had stopped our procedure till disappearance of the response waves.

We considered that persistence of the train response for more than 4 min denoted expected injury. Because of our strategy to preserve the functional integrity of the nerve, we decided to remove the neoplastic tissue as much as possible provided that there are no recorded waves.

In the last step, before dural closure, we tried to stimulate at the region where the nerve exits the brain stem and at the region of entry into the internal acoustic

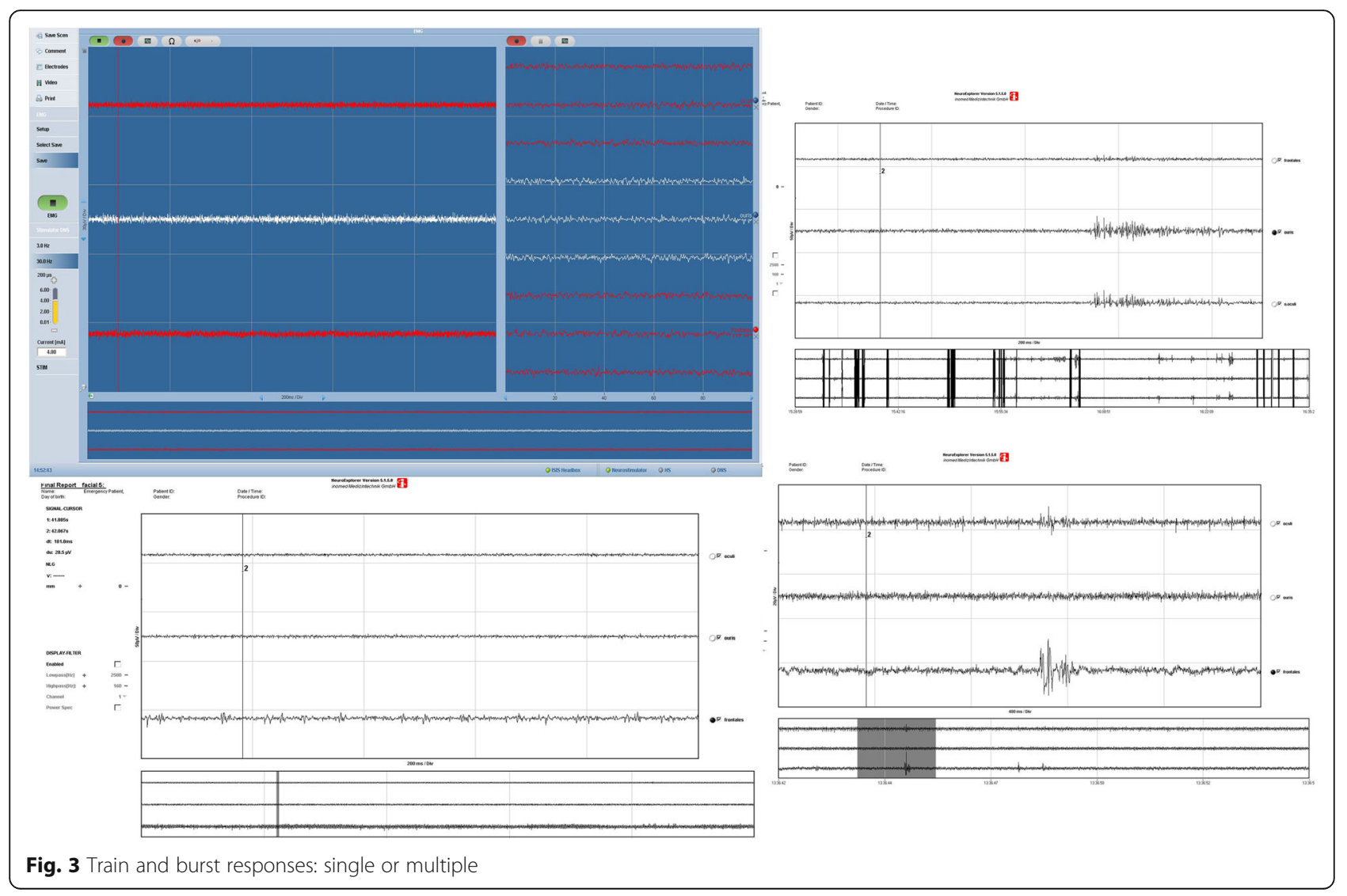


meatus. If there was a recorded response using the baseline stimulus, we expected that the nerve is functionally intact while if the intensity needed had to be increased, then weakness was expected. If no response, an injury was predicted.

\section{Postoperative care}

After recovery, a special examination of the facial nerve of the operated side was performed and, at the same time, compared with the opposite side. Regular daily examination of the nerve till the time of discharge was performed. At the time of discharge, a regular monthly follow-up was planned with the patient for 24 months. Postoperative MRI with and without contrast was requested 1 month after surgery for re-evaluation and the necessity of postoperative radiosurgery. We classified the extent of resection in our patients into two categories: near-total removal, where a small fragment of the tumor tissue was left at the internal acoustic canal region where there was a danger of injury of the facial nerve as a result of any trial of manipulation at this region (Fig. 4), and subtotal removal, where an additional amount of tumor tissue was left at the region of the cerebellopontine angle cistern (Fig. 5).

\section{Results}

This study was conducted on 32 patients with vestibular schwannomas. Total excision was an aim for all of them. Near-total excision was achieved in 20 patients $(62.5 \%)$. Subtotal excision was achieved in 12 patients (37.5\%; Table 1 ).

According to House-Brackmann (HB) grading scale, the postoperative facial nerve functions among our patients were grade I in 22 patients (68.75\%), grade III in 2 patients $(6.25 \%)$, grade IV in 4 patients $(12.5 \%)$, and grade VI in 4 patients $(12.5 \%)$. It is to be mentioned that one of our patients had immediate postoperative HB grade III improved to grade I few days postoperatively while another patient had
HB grade IV worsened to grade VI on the third postoperative day (Table 2).

Based upon the results of intraoperative neurophysiological monitoring and then the findings of the FIESTA, we found that in 30 of our patients $(93.75 \%)$, the location of the facial nerve was anterior (with detected superior and inferior fibers by intraoperative stimulation). In 10/30 patients, stimulation of the superior and inferior capsule resulted in an action potential record for a single or two muscle groups, e.g.. frontalis, orbicularis oculi, or orbicularis oris (Fig. 2). There were no recorded responses after stimulation of both superior and inferior capsule in 20/30 patients. We could not confirm the anterior location preoperatively, but two patients' FIESTA study predicted a posterior location. Intraoperatively, stimulation of the posterior capsule resulted in an action potential record for all muscle groups in separate bundles.

In patients with anteriorly located facial nerve (30/32), near-total resection could be achieved in 20/30 (66.7\%) and subtotal resection in 10/30 (33.3\%). In patients with posteriorly located nerve $(2 / 32)$, subtotal resection was achieved in all of them. There was a statistically significant difference between the anterior and posterior location of the nerves. Near-total resection was higher in the anteriorly located nerve $\left(P=0.001^{*}\right)$ while subtotal resection was higher in the posteriorly located nerve $\left(P=0.021^{*}\right.$; Table 3$)$.

Near-total resection was achieved in $20 / 32$ patients (62.5\%), and HB grade I could be achieved in $12 / 20$ patients $(60 \%)$. HB grades between II and VI could be achieved in the other 8 patients $(8 / 20,40 \%)$. In those 8 patients, the trial of dissection of the neoplasm from the nerve at or near the region of the internal acoustic meatus resulted in train activity that was prolonged (more than $2 \mathrm{~min}$ ) even after stoppage of the surgical manipulation. In the remaining 12 patients $(12 / 32,37.5 \%)$, in whom

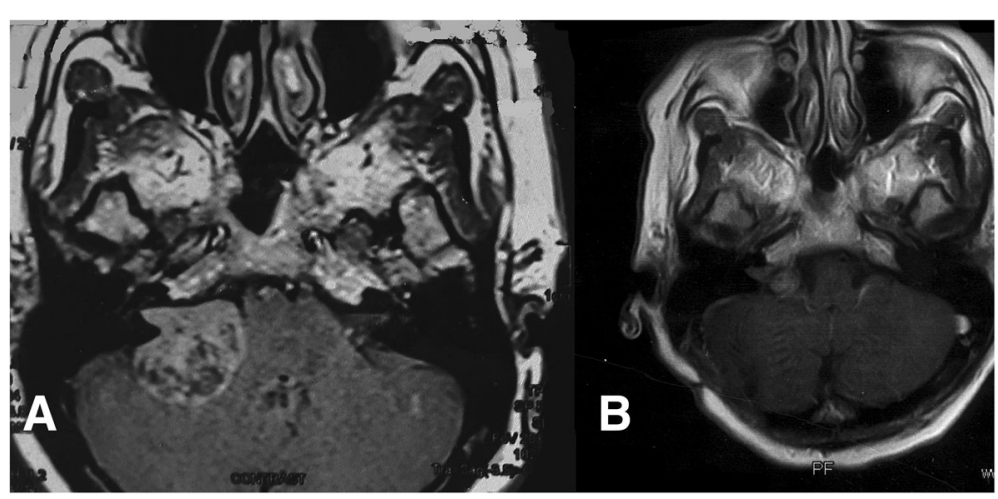

Fig. 4 a Axial T1 postcontrast MRI showing a vestibular schwannoma. b A 6-month postoperative postcontrast axial T1 MRI showing near-total resection and $\mathrm{HB}$ grade I 


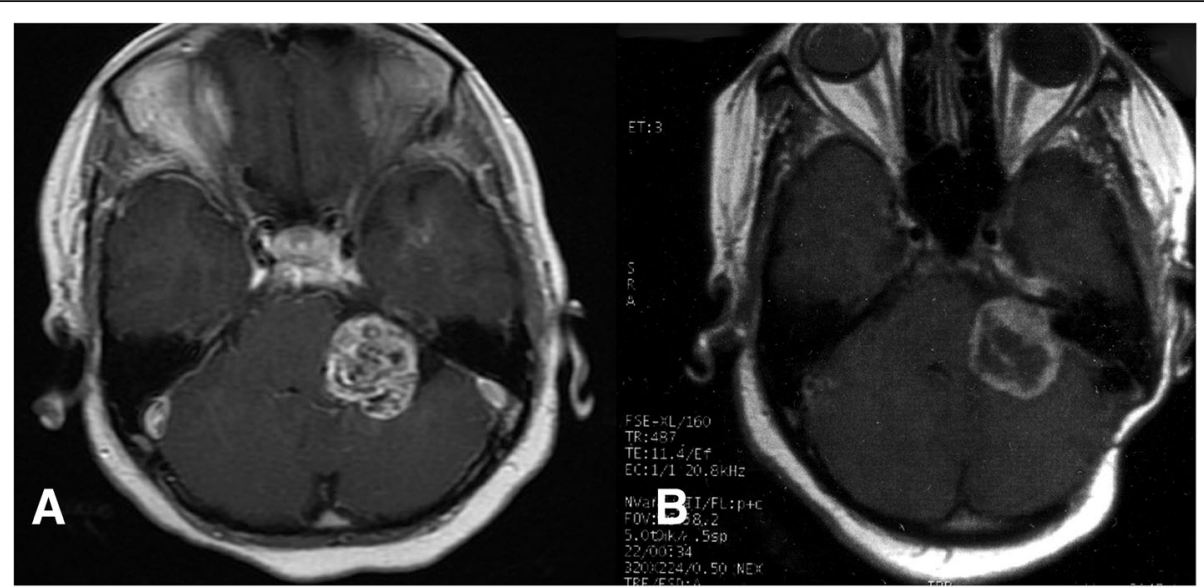

Fig. 5 a Axial T1 postcontrast MRI showing vestibular schwannoma. b An 8-month postoperative axial postcontrast MRI after radiosurgery. HB was grade I. The facial nerve was located posteriorly (Fig. 1b, c)

subtotal resection was achieved, 10 of them 10/12 (83.3\%) could achieve HB grade I while the other 2 patients $(2 / 12,16.7 \%)$ had $\mathrm{HB}$ grade that was less than $100 \%$ (Table 4 ).

There was a statistically significant difference in the functional preservation of the facial nerve between neartotal and subtotal resection where $100 \%$ preservation was higher with subtotal resection $(P=0.021)$ while less than $100 \%$ preservation of function was higher with near-total resection $(P=0.035)$.

In this study, the tumor consistency was soft in 18 patients $(18 / 32,56.25 \%)$ and suction alone was enough without hazardous traction to the capsule, so $100 \%$ preservation of function was found in $16 / 18$ patients (89\%) while less than $100 \%$ preservation of function was found in only 2 patients $(2 / 18,11 \%)$.

The tumor consistency was firm in 14 patients $(14 / 32,43.75 \%)$ in which suction alone was not enough. The dissection was accompanied by recorded train waves. So, $100 \%$ preservation was found in $6 / 14$ patients $(43 \%)$ while less than $100 \%$ preservation was achieved in $8 / 14$ (57\%).

There was a statistically significant difference in the functional preservation of facial nerve between soft and firm tumor consistency as $100 \%$ preservation of function was higher in soft consistency $(P=0.031)$ and less than $100 \%$ preservation was higher in firm consistency $(P=0.022$; Table 5$)$.

Table 1 Extent of resection

\begin{tabular}{lll}
\hline Type of removal & Number & Percentage \\
\cline { 2 - 3 } Near-total & 20 & 62.5 \\
Subtotal & 12 & 37.5 \\
Total & 32 & 100 \\
\hline
\end{tabular}

\section{Discussion}

In our hospital, FIESTA was the available imaging modality besides MRI. We have suspected the possibility of posterior location of the nerve in two patients according to the CSF signal between the nerve and the tumor especially its medial side [6] and fig [1]. Savardekar et al. [11] reported that preoperative diffusion tensor imaging with fiber tracking for facial nerve identification is a useful diagnostic tool before surgery for masses larger than 2.5 $\mathrm{cm}$. Facial nerve course was identified in $96.6 \%$ of their patients; however, the intraoperative confirmation was in $90.6 \%$ of their cases. The fallacies of that technique are affected by large masses which result in compression of the nerve and thinning of its fibers. They reported that the use of FIESTA could improve the accuracy of facial mapping. Both Chen et al. [12] and Olson et al. [13] also emphasized that FIESTA is one of the best radiologic tools that diagnose CPA masses. The nearby cranial nerves could be possibly visualized unless very large tumors. Zhang et al. [6] reported that through FIESTA study, it is possible to see the facial nerve in the cisternal segment provided that there is a CSF signal between the nerve and the neoplasm.

Table 2 Postoperative facial nerve functional outcome according to House-Brackmann grading scale

\begin{tabular}{lll}
\hline House-Brackmann grade & Number & Percentage \\
\hline HB grade I (100\%) & 22 & 68.75 \\
HB grade II (80\%)* & 0 & 0 \\
HB grade III (60\%) & 2 & 6.25 \\
HB grade IV $(40 \%)^{*}$ & 4 & 12.5 \\
HB grade V (20\%)* & 0 & 0 \\
HB grade VI $(0 \%)^{*}$ & 4 & 12.5 \\
Total & 32 & 100 \\
\hline
\end{tabular}

*Less than 100\% preservation means HB grading scale between II and VI 
Table 3 Relation between the extent of resection and the location of the facial nerve in relation to the capsule

\begin{tabular}{llll}
\hline $\begin{array}{llll}\text { Extent of } \\
\text { resection }\end{array}$ & \multicolumn{2}{l}{ Location of facial nerve } & $P$ \\
\cline { 2 - 3 } & Anterior & Posterior & \\
\hline Near-total & $20(66.7 \%)$ & $0(0.0 \%)$ & $0.001^{*}$ \\
Subtotal & $10(33.3 \%)$ & $2(100 \%)$ & $0.021^{*}$ \\
Total & 30 & 2 & 32 \\
\hline
\end{tabular}

*Statistically significant difference

In our study, we found that in 30 of our patients (93.75\%), the location of the facial nerve was anterior, anterosuperior, and anteroinferior to the capsule. The abovementioned findings were suggestive of splayed course of the nerve, where some bundles were anterosuperior while others were anteroinferior.

On the other hand, two of our patients had FIESTA study predicted a posterior location of the nerve. Actually, we found that intraoperative stimulation of the posterior capsule resulted in action potential response to the three muscle groups but in three separate bundles. We were obliged to incise the capsule in the narrow corridor between those bundles, and then, intracapsular removal of the neoplasm was done. Samii et al. [14], Sung et al. [15], and Ojemann [10] supported this opinion. (Fig. 1).

Sanna et al. [16] reported that the facial nerve is stretched and difficult to visualize but it is possible to stimulate the facial nerve proximal to the lesion. Those findings are obvious in large tumors. In our work, all of our patients had grade IV tumors. Loftus et al. [2], Wolfgang et al. [6], Kulwin and Gadol [17], and Prakash et al. [18] emphasized that the facial nerve is divided into many bundles that are stretched around the capsule and dissection of the nerve off the capsule is very risky. In our study, we had the same observation because during intraoperative mapping, we found a posterior location in 2 patients and anterior, anterosuperior, or anteroinferior in the remaining 30 . The action potential response in the three muscle groups together could be achieved either near the region of the internal acoustic meatus or at the exit of the nerve from the brain stem. In our work patients with anteriorly located facial nerve (30/32), we could achieve

Table 4 Relation between the extent of resection and the functional preservation of the facial nerve

\begin{tabular}{llll}
\hline Extent of & \multicolumn{2}{l}{ Facial nerve preservation } & Total \\
\cline { 2 - 3 } resection & $100 \%$ preservation & Less than 100\% preservation & \\
\hline Near-total & $12(60 \%)$ & $8(40 \%)$ & 20 \\
Subtotal & $10(83.3 \%)$ & $2(16.7 \%)$ & 12 \\
$P$ & $0.021^{*}$ & $0.035^{*}$ & 32 \\
\hline
\end{tabular}

Less than $100 \%$ preservation means HB grading scale between II and VI *Statistically significant difference near-total resection in $20 / 30(66.7 \%)$ and subtotal resection in $10 / 30(33.3 \%)$. In patients with posteriorly located nerve (2/32), subtotal resection was achieved in all of them. There was a statistically significant difference between the anterior and posterior location of the nerves. Near-total resection was higher in the anteriorly located nerve $\left(P=0.001^{*}\right)$ while subtotal resection was higher in the posteriorly located nerve $\left(P=0.021^{*}\right)$.

Our study concluded that the subtotal resection was associated with better facial preservation (HB grade I), $83 \%$ (Table 4). There was a statistically significant difference between the extent of resection and the functional preservation of the facial nerve $(P=0.021 \%)$ where better outcome was better with subtotal resection. Kulwin and Gadol [17] have $70-75 \%$ function preservation and $97-100 \%$ excision; the difference in our results and those of Kulwin and Gadol, especially for our patients who underwent near-total resection, is that in all of our patients, the size of the neoplasm was grade IV and the nerve was adherent to the capsule especially near the entrance to the internal acoustic meatus. This was a reason for not trying to drill the meatus for attacking the part of the neoplasm that originated inside it where in all of our patients, stimulation at the region of the internal meatus resulted in a train activity and disappeared after stoppage of procedure. Hadjipanayis et al. [19], Gurgel et al. [20], Starnoni et al. [21], and Hoshide et al. [22] also matched our opinion and advised postoperative radiosurgery.

Sun et al. [4] reported that large-sized tumors have lack of the nerve-capsule interface; moreover, elevated stimulation threshold is of bad ominous sign regarding the postoperative facial nerve function. This opinion is convenient, and we would like to add that in our work, the percentage of subtotal removal was $37.2 \%$, the possible reasons were the same as Sun et al.

One of the factors that helped us in near-total removal $(62.5 \%)$ was the soft tumor consistency, where the functional preservation was higher with soft masses $(P=$ 0.031) while lower with firm masses $(P=0.022)$ (Table 5). Samii et al. [23] reported that the predictive factors for facial nerve preservation are tumor size, cystic consistency, previous surgery or radiosurgery, and a surgeon's operative experience. Mehrotra et al. [24] found that facial preservation was better (95\%) with cystic lesions because of myxoid degeneration.

We have an opinion to preserve the facial nerve in patients having firm tumors in which subtotal excision and radiosurgery might be an option. The challenge in patients who have larger sized masses is that when subtotal removal is the solution with preservation of the facial nerve function, the residual lesion should not be larger than the size accepted for radiosurgery otherwise maximizing the size of the excised mass may be at the expense of the facial nerve outcome. 
Table 5 Relation between the consistency of the neoplasm and functional preservation of the facial nerve

\begin{tabular}{|c|c|c|c|}
\hline \multirow{2}{*}{$\begin{array}{l}\text { Consistency of } \\
\text { tumor }\end{array}$} & \multicolumn{2}{|c|}{ Facial nerve preservation } & \multirow[t]{2}{*}{ Total } \\
\hline & $100 \%$ preservation & Less than $100 \%$ preservation & \\
\hline Soft consistency & $16(89 \%)$ & $2(11 \%)$ & 18 \\
\hline Firm consistency & $6(43 \%)$ & $8(57 \%)$ & 14 \\
\hline$P$ & $0.031^{*}$ & $0.022^{*}$ & 32 \\
\hline
\end{tabular}

*Statistically significant difference

It is to be mentioned that one of our patients was a female that suffered from facial palsy after surgery (grade III). We attributed this to the trial of dissection at the region of the internal acoustic meatus. After nimodipine, she got improvement. One month later, her facial function became HB grade I. Van Gompel et al. [25] recommended that patients for whom nimodipine (or with the addition of hydroxyethyl starch) be considered perioperatively might have a chance for improvement of postoperative facial nerve function. On the other hand, one of our patients had an immediate facial palsy (HB grade IV) after surgery, and on the second postoperative day, he had worsening of the orbicularis oculi from HB grade IV to VI. Samii and Gerganov [26] attributed that the possible reason for delayed facial palsy after surgery is either vasospasm or dehydration. Hence, we recommend the use of nimodipine and hydroxyethyl starch as mentioned before.

Finally, our opinion is that not only the preoperative prediction of the course of the nerve is sufficient for functional preservation but also the degree of adherence of the nerve to the capsule, that is dependent upon the nerve-capsule interface, and the consistency of the mass. It is worthy to mention that Samii and Matthies [27] could achieve 93\% anatomical facial preservation. They also reported that the most critical point of dissection of the facial nerve is the region of entry of the internal acoustic meatus and here is the vital role of the neuromonitoring. Of $93 \%$ anatomical preservation, $51 \%$ has HB grade I, while $45 \%$ grade II to V and $4 \%$ grade VI. They also reported that the larger the tumor size, the more the adherence and hence the more difficult the postoperative functional preservation.

The limitations of this study are being retrospective and having a limited number of patients, and the diffusion tensor tractography-fiber tracking of the cranial nerves is costly and not available in our institute.

\section{Conclusion}

Functional preservation of the facial nerve postoperatively requires preoperative prediction of the location of the nerve in relation to the capsule, extra-arachnoidal dissection during surgery, and subtotal resection then radiosurgery as an alternative option if the intraoperative neurophysiological monitoring predicts a high risk and lastly the use of vasoactive medication postoperatively.

\section{Abbreviations}

CPA: cerebellopontine angle; FIESTA: fast imaging employing steady-state acquisition; HB: House-Brackmann; MRI: magnetic resonance imaging

\section{Acknowledgements}

The authors sincerely thank El-Mahalawy M and all of the department members of Neurosurgery, Tanta University Hospital, for their help in doing this work. Special thanks to Elshafey $\mathrm{R}$ of the radiology department for her cooperation.

\section{Authors' contributions}

All authors equally participated in the operation of the patients, data collection, data analysis, and scientific writing. All authors read and approved the final manuscript.

\section{Funding}

There was no funding for this study.

\section{Availability of data and materials}

The datasets used and/or analyzed during the current study are available from the corresponding author on reasonable request.

\section{Ethics approval and consent to participate}

Approved by the research ethics committee of faculty of medicine at Tanta University (reference number: 32806 obtained on 26 December 2018).

Consent for participation was not applicable as this is a retrospective study, so the ethics committee waived the consent to participate.

\section{Consent for publication}

Not applicable.

\section{Competing interests}

The authors declare that they have no competing interests.

Received: 29 January 2019 Accepted: 1 August 2019

Published online: 07 August 2019

\section{References}

1. Matthew L, Kircher MD, Jack M, Kartush MD. Pitfalls in intraoperative nerve monitoring during vestibular schwannoma surgery. Neurosurg Focus. 2012;33(3):E5.

2. Loftus CM, Biller J, Baron EM. Intraoperative neuromonitoring. 2014;24: $261-72$.

3. Nagasawa TOH, DT FBM, Trang A, Gopen Q, Parsa AT, Isaac Y. Intraoperative neuromonitoring techniques in the surgical management of acoustic neuromas. Neurosurg Focus. 2012;33(3):E6.

4. Sun MZ, Safaee M, Kaur G, Parsa AT. Neuroanatomical correlation between House-Brackmann grading system in microsurgical treatment of vestibular schwannoma. Neurosurg Focus. 2012;33(3):E7.

5. Kondziolka DS, Pirris S, Lunsford L. Improving the informed consent process for surgery. Neurosurg. 2006;58(6):1184-9.

6. Koos WT, Matula C, Lang J. Atlas of microneurosurgery of acoustic neurinomas; 2002. p. 2-88.

7. Zhang Y, Mao Z, Wei P, Jin Y, Ma L, Zhang J, et al. Preoperative prediction of location and shape of facial nerve in patients with large vestibular schwannomas using diffusion tensor imaging-based fiber tracking. World Neurosurg. 2017;99:70-8.

8. Tator $\mathrm{CH}$, Pirouzmand F. Hearing preservation and electrophysiological monitoring in the sub-occipital approach to acoustic neuromas. In: Andrew 
HK, Peter MB, editors. Operative neurosurgery. 1st ed: Churchill Livingstone; 2000. p. 873-87.

9. Wilberger J, Schurman G, Lobaugh P. Intraoperative neurophysiological monitoring. In: Tindall GT, Paul CR, Daniel BL, editors. The practice of neurosurgery: William and Wilkins; 1997. p. 451-64.

10. Ojemann RG. Suboccipital approach to acoustic neuroma. In: Andrew HK, Peter MB, editors. Operative neurosurgery. 1st ed: Churchill Livingstone; 2000. p. 864.

11. Savardekar AR, Patra DP, Thakur JD, Narayan V, Mohammed N, Bollam P, Nanda A. Preoperative diffusion tensor imaging-fiber tracking for facial nerve identification in vestibular schwannoma: a systematic review on its evolution and current status with a pooled data analysis of surgical concordance rates. Neurosurg Focus. 2018;44(3):E5.

12. Chen DQ, Quan J, Guha A, Tymianski M, Mikulis D, Hodaie M. Threedimensional in vivo modeling of vestibular schwannomas and surrounding cranial nerves with diffusion imaging tractography. Neurosurgery. 2011;68:1077-83.

13. Olson JJ, Kalkanis SN, Ryken TC. Congress of neurological surgeons systematic review and evidence-based guidelines on the treatment of adults with vestibular schwannomas: executive summary. Neurosurg. 2018:82(2):129-34.

14. Samii M, Tatagiba M, Matthies C. Acoustic neurinoma in the elderly: factors predictive of postoperative outcome. Neurosurg. 1992;31(4):615.

15. Sung KH, Dong GK, Sun HP, Chae-Yong K, Min KK, Je GC, Hee-Won J. Aggressive vestibular schwannomas with postoperative rapid growth: clinicopathological analysis of 15 cases. Neurosurgery. 2002;51(6):1381-91.

16. Sanna M, Fernando M, Russo A, Taibah A, Falcioni M, Trapani G. Atlas of acoustic neuroma microsurgery. 2nd ed; 2011. p. 52-6.

17. Kulwin $\mathrm{CH}$, Gadol AAC. Technical nuances of resection of giant (> 5 $\mathrm{cm})$ vestibular schwannomas: pearls for success. Neurosurg Focus. 2012;33(3):E15.

18. Prakash Sampath MD, David Rini MFA, Long DM. Microanatomical variations in the cerebellopontine angle associated with vestibular schwannomas (acoustic neuromas). Neurosurg Focus. 1998;5(3):E1.

19. Hadjipanayis CG, Carlson ML, Link MJ, Rayan TA, Parish J, Atkins T, Asher AL, Dunn IF, Corrales CE, Van Gompel JJ, Jeffrey MS, Olson J. Congress of neurological surgeons systematic review and evidence-based guidelines on surgical resection for the treatment of patients with vestibular schwannomas. Neurosurgery. 2018;82(2):E40-3.

20. Gurgel RK, Dogru S, Amdur RL, Monfared A. Facial nerve outcomes after surgery for large vestibular schwannomas: do surgical approach and extent of resection matter? Neurosurg Focus. 2012;33(3):E16.

21. Starnoni D, Daniel RT, Tuleasca C, George M, Levivier M, Messerer M. Systematic review and meta-analysis of the technique of subtotal resection and stereotactic radiosurgery for large vestibular schwannomas: a "nervecentered" approach. Neurosurg Focus. 2018;44(3):E4.

22. Hoshide R, Faulkner H, Teo M, Teo C. Keyhole retrosigmoid approach for large vestibular schwannomas: strategies to improve outcomes. Neurosurg Focus. 2018;44(3):E2.

23. Samii M, Gerganov V, Samii A. Improved preservation of hearing and facial nerve function in vestibular schwannoma surgery via the retrosigmoid approach in a series of 200 patients. J Neurosurg. 2006;105(4):527-35.

24. Mehrotra N, Behari S, Pal L, Banerji D, Sahu RN, Jain VK. Giant vestibular schwannomas: focusing on the differences between the solid and the cystic variants. Br J Neurosurg. 2008;22(4):550-6.

25. Van Gompel J, Agazzi S, Carlson ML, Adewumi DA, Hadjipanayis CG, Uhm $\mathrm{JH}$, Olson $\mathrm{JJ}$. Congress of neurological surgeons systematic review and evidence-based guidelines on emerging therapies for the treatment of patients with vestibular schwannomas. Neurosurgery. 2018;82(2):E52-4.

26. Samii M, Gerganov V, editors. Surgery of cerebellopontine lesions; 2013. p. 254

27. Samii M, Matthies C. Management of 1000 vestibular schwannomas (acoustic neuromas): surgical management and results with an emphasis on complications and how to avoid them clinical study. Neurosurgery. 1997; 40(1):11-23.

\section{Publisher's Note}

Springer Nature remains neutral with regard to jurisdictional claims in published maps and institutional affiliations.

\section{Submit your manuscript to a SpringerOpen ${ }^{\circ}$ journal and benefit from:}

- Convenient online submission

- Rigorous peer review

- Open access: articles freely available online

- High visibility within the field

- Retaining the copyright to your article

Submit your next manuscript at $\boldsymbol{\nabla}$ springeropen.com 